\title{
Method for geometric distortion correction in fMRI based on three echo planar phase images
}

\author{
L. Valkovic ${ }^{1}$, C. Windischberger ${ }^{2,3}$ \\ ${ }^{1}$ Institute of Measurement Science, Slovak Academy of Sciences, Dubravska cesta 9, 841 04, Bratislava, Slovakia \\ ${ }^{2}$ MR Center of Excellence, Medical University of Vienna, Lazarettgasse 14, A-1090 Vienna, Austria \\ ${ }^{3}$ Center for Biomedical Engineering and Physics, Medical University of Vienna, Austria
}

Functional magnetic resonance imaging (fMRI) is a quickly developing method for non-invasive dynamic brain studies. It uses swift acquisition sequences like echo planar imaging (EPI) that are very sensitive to susceptibility artifacts. These artifacts relate to magnetic field inhomogeneities and may cause geometric distortions. Many methods for correcting these distortions are currently used. Most common are the field mapping methods that use the map of field strength. To create a field map, different approaches can be used and different data must be acquired for each method. This paper compares a commonly used conventional gradient echo (GE) field mapping method with a 3EPI phase images based method. Although the EPI method is more sophisticated and was expected to perform better, the GE field maps showed better results in distortion correction. The cause of this is not in the method's principle itself, but in its high requirements.

Keywords: Field map, distortion correction, fMRI

\section{INTRODUCTION \& BACKGROUND}

$\mathrm{M}$ AGNETIC RESONANCE IMAGING (MRI) is a powerful tool for medicine diagnostics. A special place among MRI methods is held by functional MRI (fMRI), a key modality to perform non-invasive activation studies of the brain. The activation maps are mostly generated by statistical analysis of voxel intensity changes between stimulus and rest images of echo planar imaging (EPI) time series data. Many artifacts may occur in the data sets, because these intensity changes are typically very small $(1-4 \%),[1]$. Most severe is the geometric distortion caused by head motion or magnetic susceptibility interface.

In fMRI, head motion does not usually reduce the spatial SNR at any given time point, but may significantly reduce functional SNR, [2]. Smaller movements can be corrected during data preprocessing but larger movements that are more common, may corrupt the data completely. That is why the geometric distortion due to head motion is rather prevented than corrected during preprocessing.

In the mid and lower brain regions near the air-tissue and bone-tissue interfaces arises the problem of static magnetic field inhomogeneities. This may cause spatial and intensity distortions since tissue localization is determined by the transverse phase of the nuclear spins under applied linear gradient fields. The spins' rate of precession is proportional to the local static magnetic field strength, for example in a $3 \mathrm{~T}$ scan, the inhomogeneous static field-induced voxel shifts can easily be a centimeter or more near the sinuses. This can cause approximately 10 or more voxels of tissue to be bunched into one single voxel, [3]. These distortions are impossible to be prevented and must be corrected during data preprocessing.

This work discusses two different types of field mapping approaches and their possible use for distortion correction of the fMRI data. First is the traditional gradient echo (GE) field map and the second one is a 3EPI based field map designed by [4].
Algorithms for both approaches were designed and the methods were tested on measured phantom and human subject data. The efficiency and credibility of correction results are consequently discussed

\section{A. Echo planar imaging (EPI)}

Echo planar imaging is a rapid imaging technique, capable of capturing moving organs like heart and is very useful in dynamical imaging for brain activation analysis.

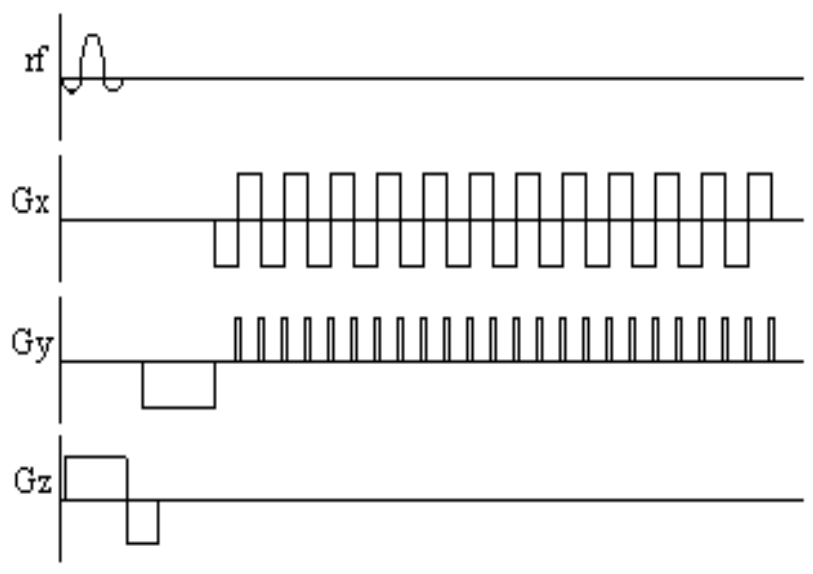

Fig.1. Pulse sequence diagram of EPI, [5]

After each excitation more than just one echo can be measured. This is done by changing the phase encoding gradient really swiftly, while the frequency readout gradient is turned on, what can be seen in Fig.1. The axes of gradient fields are labeled accordingly Gx (frequency encoding), Gy (phase encoding) and $\mathrm{Gz}$ (slice selecting).

The k-space can be filled after just one radiofrequency pulse, when using the EPI sequence. The k-space trajectory is shown in Fig.2. The strongest echo is always acquired in the center of the $\mathrm{k}$-space. 


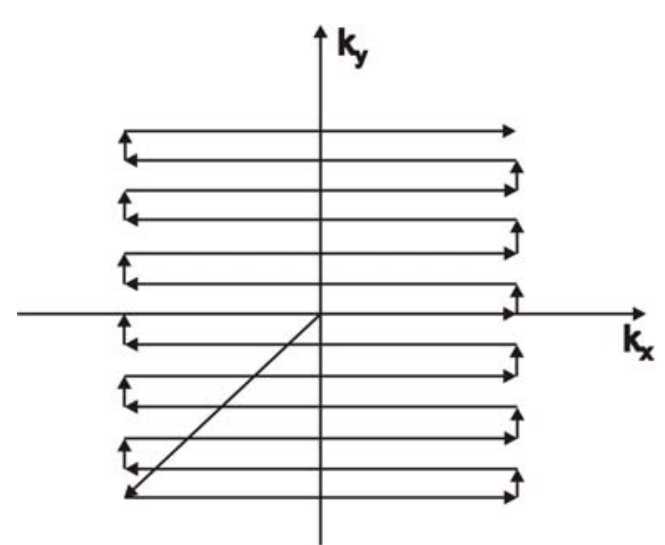

Fig.2. The k-space trajectory of EPI sequence

\section{B. EPI geometric distortion}

Brain EPI data are mostly reconstructed without correction of field inhomogeneities, hence geometric distortions are observed. These distortions appear mainly at the boundaries of regions with significant magnetic susceptibility differences. In EPI, field inhomogeneity causes pixel shifts and under severe conditions may also lead to signal loss. A long readout time makes the system more sensitive for geometric distortions, mainly in phase encoding direction, [6]-[8]. These shifts depend on EPI slice readout time and the point field inhomogeneity.

\section{Field maps}

Since geometric distortions relate to field inhomogeneities, using a map of magnetic field strength in a reverse process is a logical outcome, [9]. Field inhomogeneity $(\Delta \mathrm{B})$ relates to the phase change in time according to equation

$$
\Delta B=-\frac{\Delta \Phi}{\gamma \Delta T E}
$$

Where $\Delta \Phi$ is the phase difference, $\gamma$ is gyromagnetic ratio and $\triangle \mathrm{TE}$ is difference in echo times.

Two or more phase images must be acquired at different echo times (TE), but the differences between the individual TEs should be small enough to avoid phase-wrapping, otherwise a phase-unwrapping operation is required.

\section{GE field map}

GE field map is a commonly used type of field map usually generated by the scanner automatically and immediately after data acquisition. It is usually computed from two GE phase images scanned at different echo times and the magnitude images are used to create a brain mask. Phase-unwrapping is a standard procedure also done automatically by the scanner. As GE field map is anatomically and geometrically correct, it must be distorted the same way as the EPI data first, before it can be used to correct the EPI data. The other drawback of this approach may be the longer acquisition time and possible artifacts from the necessary distortion. While full EPI image of the human brain takes only a few seconds, the conventional GE image requires several minutes, [3].

\section{E. EPI field map}

Field maps calculated from EPI images are already distorted the same way as the rest of the data. This makes the correction procedure easier and, more importantly, it takes much shorter time scanning and the computing time is reasonable. But on the other hand these field maps must be computed manually during the data preprocessing including brain masking, phase-unwrapping, calculation of field strength and final smoothing. All methods, which use EPI phase images to calculate field maps and are able to perform phase-unwrapping assume linear or almost linear phase change over time, [4], [10].

\section{MATERIALS AND METHODS}

\section{A. Image acquisition}

EPI image series of phantom $\left(3.75 \mathrm{~g} \mathrm{NISO}_{4} \times 6 \mathrm{H}_{2} \mathrm{O}+5 \mathrm{~g}\right.$ $\mathrm{NaCl}$ in 11 of distilled water) and subject data were measured several times with different TE subintervals, from the main interval $23 \mathrm{~ms}$ to $42 \mathrm{~ms}$ ( $\triangle \mathrm{TE}=1 \mathrm{~ms}$ ), so that many different field map estimations could be calculated. Both phase and magnitude in-vivo (resp. phantom) EPI images of k-space matrix size 96 x 96 (96 x 96), image matrix size 210 x $210(1050 \times 1050)$ and 14 slices of thickness $4 \mathrm{~mm}$ $(2.5 \mathrm{~mm})$ were acquired with the measurement parameters set as follows, $\mathrm{TR}=400 \mathrm{~ms} \quad(2000 \mathrm{~ms}), \mathrm{FOV}=210 * 210$ $(1050 * 1050)$ and the $\mathrm{BW}=260 \mathrm{pixel} / \mathrm{Hz} \quad(1580 \mathrm{pixel} / \mathrm{Hz})$. Phased array head coils with 8 and 16-channels were used to evaluate possible differences. For the first test measurement the phantom was artificially distorted in phase encoding direction by changing the shimming parameters, to simulate really big geometric distortions. As the GE field map is commonly used for distortion correction, only GE phase maps of subjects were acquired. The measurement parameters for the GE images were set as for the EPI data, only the $\triangle$ TE was $5.19 \mathrm{~ms}$.

\section{B. Field map calculated out of 3EPI images, [4]}

This method is unique by its approach to phaseunwrapping, where just 3EPI phase images with different TEs are used to calculate the phase-wraps correctly. The interval between the first and the last TE must be chosen accordingly to the field strength and also equation (2) must apply so that just one phase-wrap may occur in that interval, in our experiments $\Delta \mathrm{TE}_{1,3}$ was $3 \mathrm{~ms}$.

$$
\Delta T E_{2,3}=2 \Delta T E_{1,2}
$$

Since maximally one phase-wrap may emerge, there are only 7 possible phase evolutions for each voxel. Each of these are calculated and linearly fitted and the evolution with the best fit is chosen as the real one. This way all phase-wraps are covered and the field map can be filtered and spatially smoothed for further use.

All measurements were performed on the 3T MAGNETOM Trio (Siemens AG, Erlangen, Germany). Programs for field map calculation were developed in MATLAB (MathWorks, Natick, Massachusetts). Field maps were converted into voxel shift maps for distortion 
correction. Corrected images were compared with the structural brain images of the same subjects, with the statistical parametric mapping (SPM) program, [11].

\section{RESULTS AND DISCUSSION}

As described above, EPI magnitude and phase images were acquired, along with GE magnitude images and phase maps. Consequently, field maps were calculated from the acquired data sets. Demonstration of the 3EPI based field mapping method is shown in Fig.3. The shape of the corrected phantom image is very similar to the not distorted image, and even though the correction is not perfect, for artificial distortions the method shows good results on the phantom data.

The comparison of the two studied methods is shown on subject data in Fig.4: In the a) image is a structural axial slice of a human brain; the same brain slice, but measured using EPI scanning sequence with a visible geometric distortions is shown in the b) image; after applying the 3EPI field map for distortion correction almost no pixel shifts were performed as is shown in the c) image; the GE field map was also used and with better results, image d) shows the EPI scan after distortion correction done using the GE field map.
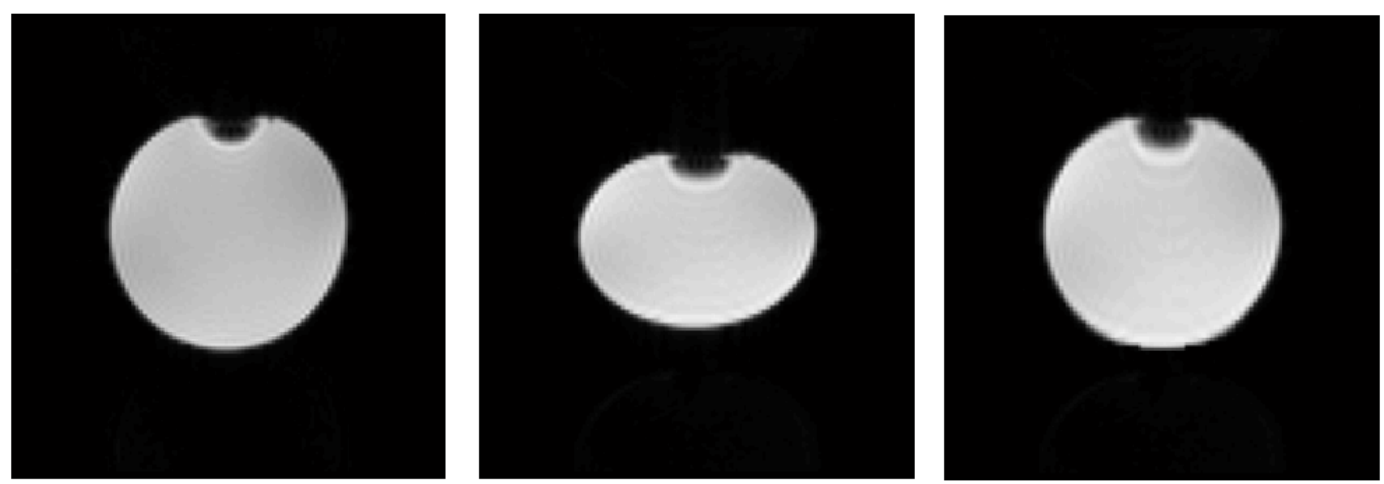

Fig.3. Demonstration of 3EPI based field mapping method on phantom images. On the left is a phantom image without distortion, image in the middle shows distorted phantom and on the right is the phantom image after distortion correction using the 3EPI based field mapping method.
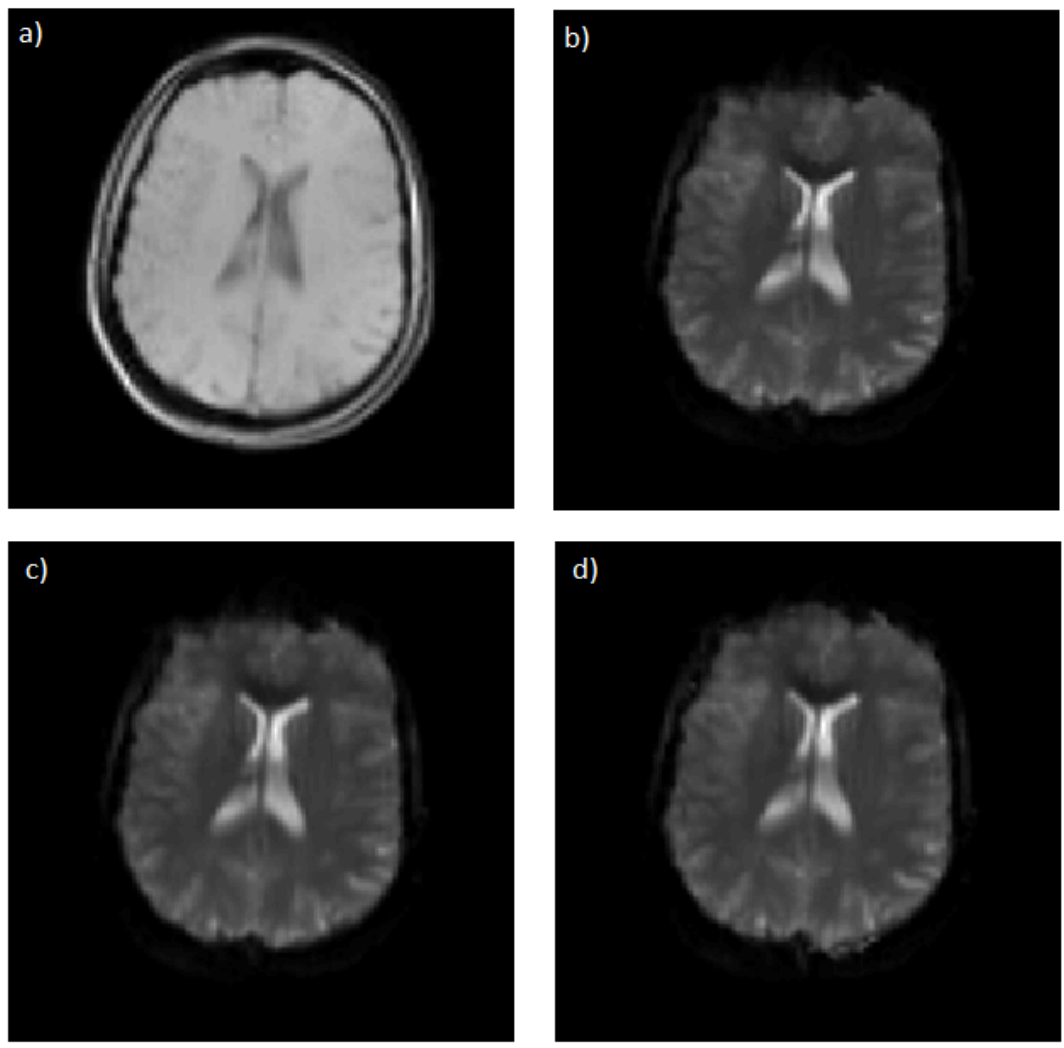

Fig.4. Comparison of EPI and GE field mapping distortion correction. This figure shows 4 axial slices of the subject brain. Image a) shows the structural image of the brain, image b) is the EPI scan of this brain. The distortion of the image is visible in the front of the brain. Field map distortion correction methods were used. In image c) is the brain after EPI field map correction and in image d) is the brain after GE field map correction. 


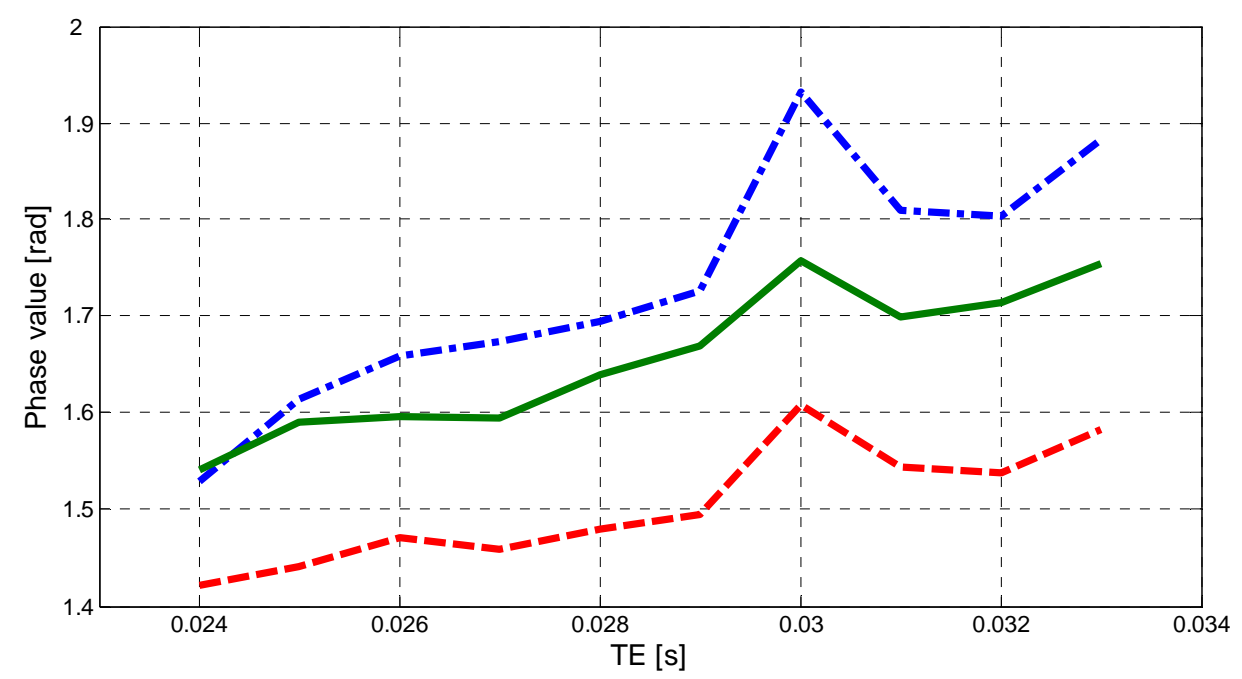

Fig.5. Phase evolution in time of three different randomly chosen voxels of the measured phantom EPI phase images. The phase does not evolve in time linearly not even almost linearly, the phase fluctuations that occur are not even the same for every voxel of the phantom.

There is a meaningful explanation why the GE based field mapping method shows better results than the 3EPI based method, as can be seen in Fig.4. The acquired data sets do not fulfill the main and essential requirement for the EPI method to work correctly. Namely the linear or almost linear phase evolution in time. Fig.5 shows the time evolution of phase for three different, randomly chosen voxels of the phantom data. The phase evolution measured is not linear, many phase fluctuations occur in the studied interval. With this kind of phase evolution, the linear fitting algorithm for phase-unwrapping is absolutely inadequate.

Field maps calculated using data sets with non-linear phase evolution in time cannot be calculated correctly. This significant miscalculation makes these magnetic field maps inefficient for geometric distortion correction. Therefore, before using any EPI based field mapping method, no matter how sophisticated its main principle is, it is highly recommended to check first if the key requirements are fulfilled.

\section{CONCLUSIONS}

Two different magnetic field mapping methods were used for geometric distortion correction. Both methods have been evaluated on acquired phantom and subject data sets. EPI field mapping method is in general more sophisticated and has many advantages, but since the phase did not evolve linearly or almost linearly, it did not show reliable results. Since the main requirement for right calculation of EPI based field map was not fulfilled, the GE field map used for the same purpose showed better results and is therefore considered to be more robust.

\section{ACKNOWLEDGMENTS}

This work was supported in part by the Tatra Bank Foundation grant for students work abroad 2008sds091 and in part by Slovak Scientific Grant Agency VEGA 2/0142/08.

\section{REFERENCES}

[1] Yeo, D.T.B., Fessler, J.A., Kim, B. (2008). Concurrent correction of geometric distortion and motion using the map-slice-to-volume method in echo-planar imaging. Magn. Reson. Imaging, 26, 703-714.

[2] Huettel, S.A., Song, A.W., McCarthy, G. (2009). Functional Magnetic Resonance Imaging. Sunderland: Sinauer Associates.

[3] Holland, D., Kuperman, J.M., Dale, A.M. (2010). Efficient correction of inhomogeneous static magnetic field-induced distortion in Echo Planar Imaging. NeuroImage, 50 (1), 175-183.

[4] Windischberger, C., Robinson, S., Rauscher, A., Barth, M., Moser, E. (2004). Robust field map generation using a triple-echo acquisition. J. Magn. Reson. Imaging, 20, 730-734.

[5] Stuart, C. (1997). Functional MRI: Methods and Applications. Doctoral thesis, University of Nottingham.

[6] Jezzard, P., Balaban, R.S. (1995). Correction for geometric distortion in echo planar images from B0 field variations. Magn. Reson. Med., 34, 65-73.

[7] Kadah, Y.M., Hu, X. (1998). Algebraic reconstruction for magnetic resonance imaging under B0 inhomogeneity. IEEE Trans. Med. Imaging, 17, $362-370$.

[8] Jezzard, P., Clare, S. (1999). Sources of distortion in functional MRI data. Hum. Brain Mapp., 8, 80-85.

[9] Moghaddam, A.N., Soltanian-Zadeh, H. (2003). Mapping of magnetic field inhomogeneity and removal of its artifact from MR images. In Medical Imaging 2003. Proceedings of SPIE, Vol. 5032, 780-787.

[10] Reber, P.J., Wong, E.C., Buxton, K.B., Frank, L.R. (1998). Correction of off-resonance related distortion in echo-planar imaging using EPI-based field maps. Magn. Reson. Med., 39, 328-330.

[11] SPM - Statistical Parametric Mapping, http://www.fil.ion.ucl.ac.uk/spm 